\title{
An observational study of post-operative Volumetric Modulated Arc Radiotherapy (VMAT) of breast cancer by immobilizing patients on All-in-one (AIOTM) solution Breast and Lung Board without usingthermoplastic mask: Risk of collusion of gantry with couch exists if acquisition of Cone-beam Computed Tomography (CBCT)is attempted for set-up verification.
}

Ramaiah Vinay Kumar ( $\sim$ vinaykumar33223@gmail.com )

Research

Keywords: Breastmalignancies, Volumetric Modulated Arc Therapy(VMAT), All-in-one (AIOTM) Solution Breast and Lung Board, Cone Beam Computed Tomography (CBCT)

Posted Date: April 30th, 2020

DOI: https://doi.org/10.21203/rs.3.rs-24920/v1

License: @ (i) This work is licensed under a Creative Commons Attribution 4.0 International License. Read Full License

Version of Record: A version of this preprint was published at Journal of Radiation Oncology on August 29th, 2020. See the published version at https://doi.org/10.1007/s13566-020-00434-y. 


\section{Abstract \\ Background:}

Automatic Cone-beam computed tomography (CBCT) based image matching for set-up verification is recommended as compared to 2-D match for postoperative local / loco-regional radiotherapy of breast cancer patients by Volumetric Modulated Arc Therapy (VMAT) technique. However, in supine position, off-midline peripheral body Clinical Target Volume (CTV) of unilateral breast cancer patients immobilized on Breast and Lung board of All-in-One (AIO) positioning systemmay necessitate augmented movement of couch in ' $x$ ' and ' $z$ ' axis thereby raising the risk of collusion of $x$-ray sources / detectors system with couch.

\section{Methods and Materials:}

VMAT was planned by a pair of partial arc for whole target volume for seven consecutive post-operative breast cancer patients (five post-mastectomy and two post-breast conservation patients). Tattoo based set-up by shift of treatment table in $x$-, $y$ - and $z$-axis as determined by Treatment Planning System followed by X-rays with planar image acquisition and online 2-D imaging matching was performed for set-up verification. In-room $360^{\circ}$ rotation of $x$-ray source and detector system of linear accelerator (linac) was performed before $x$-ray planar image acquisition.

\section{Results:}

Completion of $360^{\circ}$ rotation in-room of $x$-ray source and detector system of linacaround the machine iso-centre was not possible in six out of seven patients due to possibility of collusion of gantry with contralateral side of the couch.

\section{Conclusion:}

Performing CBCT for generating 3D images for computed tomography (CT) reconstruction may not be practical for patient set-up verification of post-operative radiotherapy of unilateral breast cancer patients positioned supine on breast and lung board.

\section{Introduction}

All-in-one (AIO) solution [The AIO ® (Orfit Industries NV, Wijnegem, Belgium)] for positioning and immobilizing patients for radiotherapy was introduced in 2005. Breast and lung board is one of the four sets of cushions for supine treatment of breast and thoracic malignancies. [1] Immobilizing post-operative breast cancer patients supine on series of cushions for radiotherapy of ipsilateral chest wall / whole breast may necessitate augmented shift of linac couch in ' $x$ ' and ' $z$ ' axis during set-up verification in order to implement iso-centric treatment. Such drastic shift of treatment couch may introduce the risk of collusion of Gantry with treatment couch if acquisition of $\mathrm{CBCT}$ is attempted given the recommendation that automatic CBCT based image matching as the optimal set-up modality for implementation of VMAT of chest wall / whole breast. [1, 2] Hence the need to study the possibilities of acquiring CBCT with post-operative breast cancer patients on-board immobilized on breast and lung board.

\section{Methods And Materials}

Two of the nine post-operative breast cancer patients defaulted for radiotherapy after computed tomography (CT) simulation. (Table 1) Therefore, caserecords of only seven patients were reviewed for data collection and analysis. Informed consent for CT simulation and VMAT was obtained from all the nine consecutive post-operative breast cancer patients presenting for radiotherapy. Confidentiality and privacy of all the patients were maintained throughout the study. Study adhered to principles of the Declaration of Helsinki 1975 (as revised in 2000). Consent was obtained for clinical photographs taken during study and utmost care was take to conceal the identity of patients. The study is outcome of real-world practice of VMAT for post-operative irradiation of chest wall / breast and regional lymph node areas. The work-flow for implementation of VMAT is as follows: a) arranging of breast and lung board on CT simulator couch b) position of patients on breast and lung board c) Ascertaining the position of patients for reproducibility d) tattooing and fidicual placement at point of three laser cross hair on skin surface .i.e. one each on anterior midline, right lateral and left lateral chest wall d) acquisition of non-contrast CT e) Importing of simulation CT and contouring of target and normal structures $\mathrm{f}$ ) VMAT planning by medical physicists $\mathrm{g}$ ) Plan approval by radiation oncologists and registration of patient details $\mathrm{h}$ ) exporting of VMAT plan for implementation on linac and scheduling the same along with AP and lateral digitally reconstructed radiograph (DRR) for set-up verification of treatment position. Simulation CT data set were also scheduled with VMAT plan for matching with CBCT based on-board imaging for set-up verification. However, planar x-ray image based matching was planned mode of set-up verification i) positioning of patients on breast and lung board following procedure similar to that for CT simulation, matching of body tattoo with laser cross-hair on linac, shifting of couch in $x-, y-, z$ - axis if needed and checking SSD j) online 2-D based image matching and final shifting of couch in $x-, y$-and $z$ - axis $k$ ) Execution of VMAT. 
Table 1

Clinical profile of patients

\begin{tabular}{|c|c|c|}
\hline \multirow[t]{2}{*}{ Age range } & \multicolumn{2}{|l|}{$30-56$ years } \\
\hline & & No. of patients \\
\hline \multirow[t]{2}{*}{ Menopausal status } & Pre-menopausal & $5^{\#}$ \\
\hline & Post-menopausal & 4 \\
\hline \multirow[t]{3}{*}{ Body habitus } & Thin & $3^{\ddagger}$ \\
\hline & Normal & $3^{\ddagger}$ \\
\hline & Obese & 3 \\
\hline \multirow[t]{3}{*}{ Stage of the disease } & Early & 2 \\
\hline & Locally advanced & $6 \#$ \\
\hline & Metastatic & 1 \\
\hline \multirow[t]{2}{*}{ Histology } & Invasive Ductal Carcinoma & 7 \\
\hline & Malignant / borderline phyllodes tumor & $2^{\#}$ \\
\hline Comorbidities & Retroviral disease & $1^{\ddagger}$ \\
\hline \multirow[t]{3}{*}{ Nature of surgery } & Modified Radical Mastectomy & 5 \\
\hline & Breast conservation surgery (BCS) & 2 \\
\hline & Simple mastectomy & $2^{\#}$ \\
\hline \multirow[t]{2}{*}{ Intent of therapy } & Post-operative & $8^{\#}$ \\
\hline & Palliative & 1 \\
\hline \multirow[t]{4}{*}{ Clinical Target Volume (CTV)* } & Chest wall alone & $2^{\#}$ \\
\hline & Chest wall and regional lymph node area & 5 \\
\hline & Whole breast & 1 \\
\hline & Whole breast and regional lymph node area & 1 \\
\hline \multicolumn{3}{|c|}{ * All patients were planned by a pair of partial arc VMAT technique with online 2-D matching of images for set-up verification. } \\
\hline
\end{tabular}

Immobilization of patients on Klarity base plate and thermoplastic immobilization mask was the clinical practice before introduction of breast and lung board immobilization. Patients were initially simulated, planned and VMAT was executed on Klarity base plate and thermoplastic immobilization mask in the transition period of changing over from one kind of immobilization to another different type of immobilization. Once the work flow for immobilization on breast and lung board was established, patients were completely switched over from klarity immobilization system with thermoplastic mask to breast and lung board immobilization system without thermoplastic mask.

Coming to the present study, nine consecutive post-operative breast cancer patients were immobilized on Breast and Lung board without thermoplastic mask. Patients were immobilized on combination of $0^{\circ}$, one of $5^{\circ} / 10^{\circ} / 15^{\circ}$ angle wedge, low or high arm rest $\left(20^{\circ}\right.$ or $30^{\circ}$ angle respectively), feet and knee cushions. Indexed head support by using one of the head rest ranging from number 1 to 6 was used to position the head in reproducible manner. A pair of grip pole mounted over grip pole collar to provide hand grip for over-head abducted arms were also utilized. $L$ shaped hand grip projecting from one of the grip pole was used in case of painful limitation of / difficulty in abduction of arm due to post-operative fibrosis. The cushion set and accessories were used with base plate that was secured to couch top using two-pin indexing bar. Patient was relaxedly positioned supine over breast and lung board with head placed over appropriate head rest fixed to slot on the board after asking them to remove all clothes and jewels till pubis. Arms were abduced over-head and positioned over arm rest with hands holding hand grip. Central laser passed exactly along midline of patients' body from root of nose through neck, thorax and abdomen to pubis. Positioning patients in this manner ensured that the same can be reproduced during set-up and treatment. No thermoplastic mask was used for immobilization of patients as the same was not procured and supplied.

Simulation was performing by spiral CT scan obtaining slice thickness of $5 \mathrm{~mm}$ (images spaced from base of skull to lower edge of liver) without contrast on Philips Brilliance BigBore ${ }^{\text {TM }}$ (Philips Medical Systems, Madison, WI). Midline laser of CT simulator was used to position the patients in straight line on the set of cushions before acquisition of scan. Three skin tattoos, two lateral and one anterior, were marked for position verification by alignment to the 3 laser system. Three $2 \mathrm{~mm}$ lead ball on $15 \mathrm{~mm}$ skin marking label [KSU-SL-20 - SureMark ${ }^{\mathrm{TM}}$, Newark, OH] was placed on tattooed laser cross-hair on anterior midline and lateral thoracic wall. Positional quality of simulation CT scan was ascertained by scrolling through the CT dataset by placing TPS crosshair over the tip of spinous process. If consecutive tip of spinous process had deviated $>2 \mathrm{~mm}$ from the TPS crosshair, then re-simulation was performed after re-aligning of 
patients' midline with midline laser by technologist / radiation oncologist. If the deviation persisted despite re-positioning, it was anticipated that the same would be reproducible during treatment. The images with DICOM 3 format were transferred to the treatment planning system (TPS) by local network. Simulation CT so transferred was imported to Monaco TPS version 5.1 (Elekta CMS, Maryland Heights, MO, USA) and RTOG consensus guidelines were used for contouring chest wall / breast and draining lymph node areas.

Medical physicists planned VMAT on simulation CT and same was approved by radiation oncologists if the plans were satisfactory with respect to target volume coverage and dose to critical normal tissue. Next step is registration of patient, scheduling of approved plan along with AP / lateral DRRs and simulation CT into Radiation Oncology Information System (interface software) MOSAIQ ® Radiation Oncology (Elekta IABS, Kungstensgatan, SE, Stockholm). During the first treatment session, the patient was aligned by the laser system with the three skin tattoos and two orthogonal 2D images (one each of EPID images and flat panel kV image acquired simultaneously) were acquired, typically anterior-posterior and latero-lateral. Tattoo-based set-up of patients immobilized on breast and lung board and online 2D image matching was performed for set-up verification before implementation of VMAT. Set-up verification was performed using orthogonal $\mathrm{kV}$ and $\mathrm{MV}$ image (flat panel detector and EPID generated $\mathrm{kV}$ and MV images by using CBCT-generated and linacgenerated $x$-ray respectively). The images were matched with the digitally reconstructed radiographs (DRRs) from the CT simulation using a dedicated software. The alignment was validated by a radiation oncologist on the basis of bone anatomy. The region of interest (ROI) of the patient for $2 \mathrm{D}$ image matching was from base of skull to lower extent of rib cage. Vertebra bodies, interface of pleura with ribs, clavicle, head, neck and upper shaft of humerus were matched in online 2D AP images. Vertebra bodies, intervertebral foramina, spinous process, manubrio-sternal joint, outer and inner cortex of sternum were matched in online 2D lateral images. Active Breathing Coordinator or 4-D CT was not utilized for simulation.

Treatment of chest wall / breast with or without draining lymph node areas was planned by VMAT to a total dose of 50 Gy in 25 fractions of 2 Gy by a pair of partial arc (one clockwise and another counter-clockwise) technique over a period of 5 weeks. The planning target volume (PTV) was obtained by a 5 mm expansion around the CTV except for the posterior margin where the PTV expansion was trimmed anticipating dose to ipsilateral lung. All patients were treated in supine position with both arm abducted over-arm and hand held hand grip fixed to grip collar on base plate. Knee joints were placed in flexed position over knee rest that was placed over ankle board to facilitate setup reproducibility.

In the entire study, tattoo-based set-up and online 2D matching was the planned and primary mode of set-up verification. However, based on in-puts from medical physicists and technologists, it was subsequently planned to check for collusion of gantry with couch by moving the gantry $360^{\circ}$ around iso-centre (simulating on-board acquisition of CBCT) in the in-room presence of two radiotherapy technologists and radiation oncologist after shifting the patients' couch in $x$-, $y$ - and $z$-axis as determined by Treatment Planning System (TPS) necessary for implementingiso-centric treatment by VMAT. All procedures followed were in accordance with the ethical standards of the responsible committee on human experimentation (institutional and national) and with the Helsinki Declaration of 1975, as revised in 2008 (5). Informed consent was obtained from all patients for being included in the study.

\section{Results}

Data of seven patients were available for analysis. Attempt at rotating gantry $360^{\circ}$ degrees around the couch (or isocentre) resulted in impending collusion of gantry with couch in six out of seven breast cancer patients immobilized in supine position on breast and lung cancer board. Gantry could move $360^{\circ}$ without collusion and could not move $360^{\circ}$ due to impending collusionafter shifting couch in $x-y$-, and $z$ - axis for set-up verification of VMAT boost of post-operative cavity in one patients each who had tumour in upper inner quadrant and had undergone Breast conservation surgery. CBCT based image matching and set-up verification was feasible upfront in only one patient and in second phase for radiotherapy boost of post-operative cavityin another patient. [Figure 1-3]

\section{Discussion}

Published literature on dosimetry and planning techniqueof VMAT in post-operative setting for local / loco-regional treatment of breast cancer patients have produced conflicting results. [3-7] Without going into details of pros and cons of VMAT, the current manuscript presents result of observational study with respect to implementation of VMAT for post-operative breast cancer patients positioned and immobilized supine on breast and lung board. AIO solution immobilization system introduced in 2005 is series of cushion set that enhances patients' comfort and treatment position reproducibility. For head and neck cancer patients, there is no cushion set and patients are immobilized on head rest placed over slot on base plate. Rest of the regions of body can be immobilized both in supine and prone position on cushion sets. However, it was acknowledge that the height of cushion set was an issue from the beginning as it may increase the risk of collusion with gantry [1] Hencecushion sets were continuously modified by decreasing the height in order to reduce the risk of collusion. Implementation of online 2D- and CBCT-based image matching for set-up verification and VMAT for treatment of midline body core and visceral tumours of patients positioned and immobilized on cushion set is carried-out in our clinic without any hassle after introduction of this system of immobilization. [Personal communication]However, radiation target of breast cancer lies in body's periphery .i.e. geometrically, Clinical Target Volume (CTV) of breast tumour lies in anterio-lateral chord of circumference of axial thoracic cage.Couch has to be moved further particularly in $\mathrm{x}$ - and z-axis (laterally and vertically respectively) with patients positioned on breast and lung board than regular treatment on baseplate alone in order to bring the centre of CTV to machine iso-centre. Augmented movement of couch takes it to the secant of circle of rotation of gantry around iso-centre thereby raising the possibility of collusion of gantry with couch. [Figure 4] This hypothesis is supported by the result of our study in that collusion of gantry with couch (whose position was corrected to take the planning iso-centre of target to machine iso-centre) is a possibility in six out of seven post-operative breast cancer patients positioned supine on breast and lung board when the attempts were made to rotate the gantry $360^{\circ}$ around its iso-centre. Such collusion could have been prevented by placing planning iso-centre inside the ipsilateral lung rather than at centre of CTV. However, concern were raised of poor quality of plan with respect to target coverage and dose to critical normal structure. Hence, present study did not alter the planning parameters in the interest of optimal dosimetry for VMAT plan.

Further, concerns were raised regarding methodology of this study. The methodology in practice is as follows: a. Position of patients on couch so that linac cross hair matches exactly the simulation crosshair $b$. Then move the patient in $x-, y-, z$-axes $c$. Returning the couch to central position / initial position of 
patient positioning d. Performing CBCT e. moving the couch to treatment position e. Image registration (superimposing CBCT image over Simulation CT image) and acceptance of anatomy $\mathrm{f}$. Delivery of fraction of radiation. All these can be performed automatically or manually. However, such methods requires ascertaining quality assurance, quality control and precision of couch movement frequently that may not be feasible in high-volume and resource-constraint set-up. This methods also negates the very purpose of CBCT in treatment iso-centric position. There are high possibilities of introduction of random and systemic errors during this type of patient set-up for radiotherapy. To test this hypothesis, we applied this set-up method to our next four consecutive patients followed by verification of anatomy by planar / orthogonal hybrid of $\mathrm{kV}$ and $\mathrm{MV} \mathrm{x}$-rays. At least $1-2 \mathrm{~mm}$ anatomical corrections were required in all the three axes of set-up in all the four patients. (Results of thespatients set-up verification by CBCT by initial methodology will be updated)

All the seven patients were treated by VMAT as the plan was performed by partial arc technique. Here a pair of partial arc (gantry moves clockwise direction and counter-clockwise during treatment in first and second partial arc respectively) are place on ipsilateral side and couch moves away from the gantry. Mounted kV CBCT source, kV flat panel detector and electronic portal imaging device (EPID) is retracted back to its base position. All the above factor rules out the possibility of collusion of gantry with couch moved to treatment position. Clinical importance of this study lies in the fact that Rossi $\mathrm{M}$ et al have recommended СВСТ based image matching for set-up verification as it improves accuracy of treatment delivered by VMAT. [2] Results of our study indicates that CBCT may not be feasible in post-operative breast cancer patients immobilized supine on current breast and lung board. To best of our knowledge, we came across only one study from Belgium by Boute B et al examining feasibility of CBCT of post-operative breast cancer patients immobilized both supine and prone on breast and lung board and prone breast board respectively. In this study, two different types of patient support devices for prone radiotherapy in crawl position after breast conservative surgery were utilized on special type of couch (I-Beam EVO couch blade of an Elekta Synergy linear accelerator). Support devices for crawl position were built as breast boards (for CT simulation) and breast couches (for treatment). Prone breast boards rest entirely on the treatment couch surface while prone breast couches replace the couch blade (or its cranial part). Patients were also treated supine on breast and lung board in this study. However, authors have only presented the dosimetric comparison between the two positions. From the published literature and results presented, it is not clear if CBCT was used for set-up verification for management of supine breast cancerpatients in the Belgian Study. [8] Unlike study published by Boute $B$ et al, breast and lung board in our study was positioned over regular carbon fibre couchof Elekta Versa HD linac and large majority of patients presented for post-mastectomy radiotherapy rather than therapy of conserved breast. With the current radiation equipment and non-availability of floor laser, concerns apart from difficulty in tattooing, positioning and reproducibility issues - were also raised regarding possible collusion of Gantry with couch if attempts were made to rotate it $360^{\circ}$ around its iso-centre if patients were immobilized on prone breast board as the height of this board was around $23 \mathrm{cms}$. The height of breast and lung board ranged between 11 and $15 \mathrm{cms}$ and we feel it is too early to comment on prone breast board at this point of time. Also, aim of our study was to assess the possibility of acquiring CBCT of post-operative breast cancer patients immobilized on breast and lung board rather than that of those immobilized on prone breast board.To summarize, immobilizing post-operative breast cancer patients on breast and lung board in supine position may lead to collusion of Gantry with couch if attempts are made to rotate gantry $360^{\circ}$ around its iso-centre (simulating acquisition of CBCT).

\section{Conclusion}

Possibilities of collusion of gantry with couch (after the couch is brought to treatment position by shifting it in $x-, y-, z-a x e s$ as determined by TPS in order to bring planning iso-centre / centre of CTV to machine iso-centre) existed in six out of seven post-operative breast cancer patients immobilized supine on breast and lung board if attempts were made to rotate gantry by $360^{\circ}$ around iso-centre. Acquisition of CBCT requires that the whole system of gantry and mounted $\mathrm{kV}$ image source of CBCT, $\mathrm{kV}$ detector and EPID freely rotates $360^{\circ}$ around the isocentre of linear accelerator. Recommendation in favour of CBCT based image matching for implementation of VMAT in post-operative management of breast cancer patients necessitates newer strategies for radiotherapy planning of breast cancer patients immobilized on breast and lung board so as to avoid collusion of gantry with couch in order to acquire and utilize CBCT for set-up verification. Hence, further studies are needed to optimizethe process of radiotherapy planning of patients immobilized on cushion sets.

\section{List Of Abbreviations}

AIO - All-in-one (AIO) solution; CBCT - Cone Beam Computed Tomography; VMAT - Volumetric Modulated Radiotherapy; CT - Computed Tomography; kV Kilovoltage, MV - Megavoltage, EPID - Electronic Portal Imaging Device; CTV - Clinical Target Volume; TPS - Treatment Planning System; 2D - two dimension, 3D - three dimension, 4D - four dimension, ROI: region of interest, DRR - Digitally reconstructed radiography, RTOG - Radiation Therapy Oncology Group; Dicom - Digial Imaging and Communications in Medicine

\section{Declarations}

Ethical Approval and Consent to participate: Ethical approval is not needed as the study presents real-world evidence. Consent of all the participants was taken prior to the study.

Consent for publication: Not applicable

Availability of supporting data: Will be made available

Competing interests: None

Funding: Not applicable

Authors' contributions: Ramaiah Vinay Kumar has contributed to conceptualization, data collection, analysis, writing of manuscript and critical analysis of the same 
Authors' information (Optional): Ramaiah Vinay Kumar, Assistant Professor of Radiation Oncology, Kidwai Memorial Institute of Oncology, Bangalore - 560029

Conflict of interest: None

\section{References}

1. Orifit - The precision expert. Press Information. Orfit Industries Received FDA 510(k) Clearance for the new AIO Solution®. Norfolk, VA, August 30, 2019. Available from URL:

https://www.astro.org/ASTRO/media/ASTRO/News\%20and\%20Publications/Press\%20Kits/PDFs/OrfitIndustriesReceivedFDA510kClearanceforthenewAll [Accessed on 28th November 2019].

2. Rossi M, Boman E, Skyttä T, Haltamo M, Laaksomaa M, Kapanen M. Dosimetric effects of anatomical deformations and positioning errors in VMAT breast radiotherapy. J ApplClin Med Phys. 2018 Sep;19(5):506-16.

3. Qi XS, Liu TX, Liu AK, Newman F, Rabinovitch R, Kavanagh B, et al. Left-sided breast cancer irradiation using rotational and fixed-field radiotherapy. Med Dosim. 2014 Autumn;39(3):227 - 34.

4. Xi D, Ding Y, Hu R, Gu W, Mu J, Li Q. Advantages of a technique using two 50 degree arcs in simultaneous integrated boost radiotherapy for left-sidebreast cancer. Sci Rep. 2017 Nov 7;7(1):14748.

5. Yu PC, Wu CJ, Nien HH, Lui LT, Shaw S, Tsai YL. Tangent-based volumetric modulated arc therapy for advanced left breast cancer. RadiatOncol. 2018 Nov 28;13(1):236.

6. Pasler M, Lutterbach J, Björnsgard M, Reichmann U, Bartelt S, Georg D. VMAT techniques for lymph node-positive left sided breast cancer. Z Med Phys. 2015 Jun;25(2):104-11.

7. Lee B, Lee S, Sung J, Yoon M. Radiotherapy-induced secondary cancer risk for breast cancer: 3D conformal therapy versus IMRT versus VMAT. J Radiol Prot. 2014 Jun;34(2):325-31.

8. Boute B, De Neve W, Speleers B, Van Greveling A, Monten C, Van Hoof T, et al. Potential benefits of crawl position for prone radiation therapy in breast cancer. J ApplClin Med Phys. 2017 Jul;18(4):200-5.

\section{Figures}




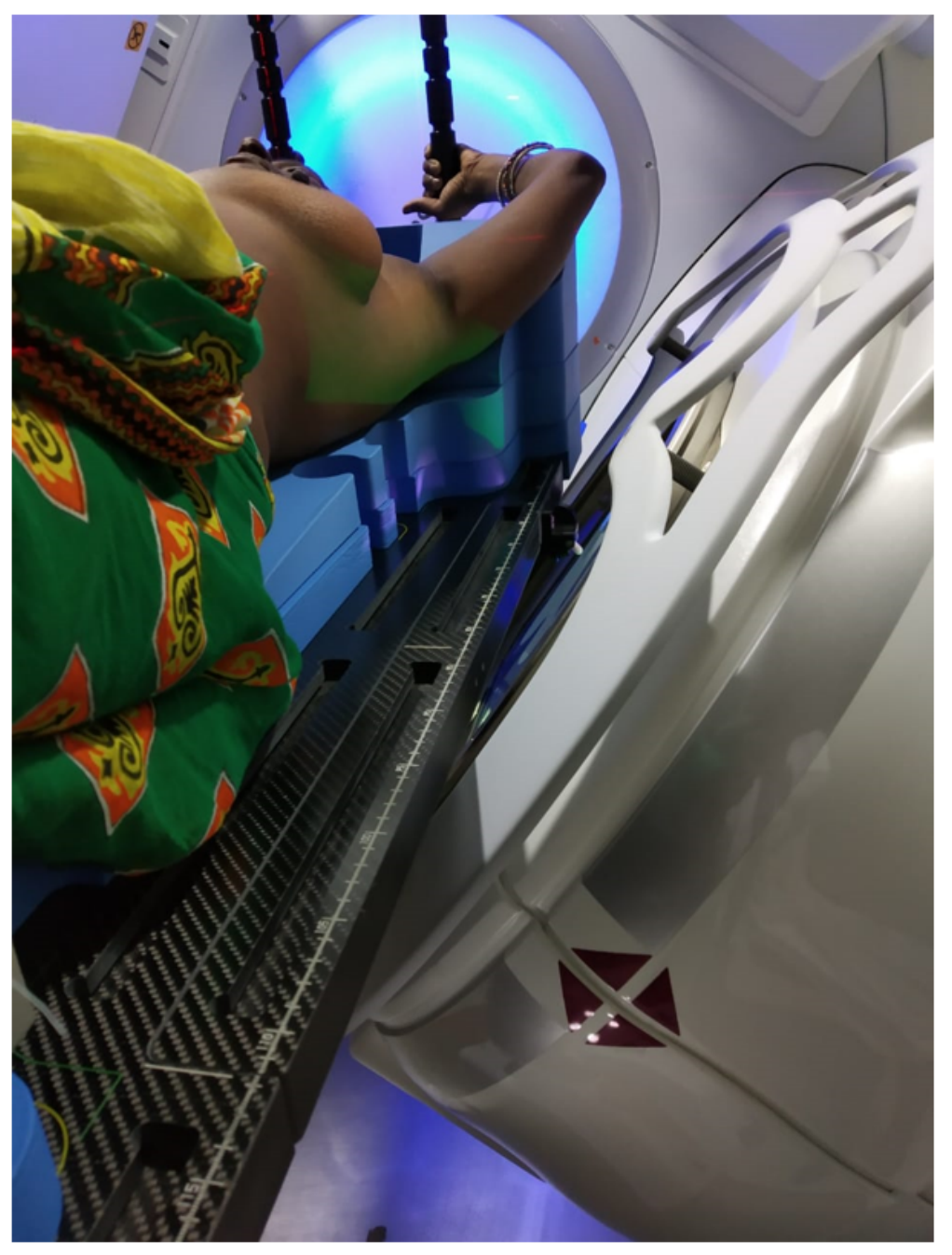

\section{Figure 1}

Immobilization of patient without thermoplastic mask over AIO breast and lung board, arms abducted over low arm rest and both hands holding a pair of grip poles placed overhead using grip collar. Figure depicts impending collusion of gantry with left side (contralateral) of couch after shifting of couch in $x$-, $y$ - and z-axis as determined by TPS for iso-centric treatment of chest wall and draining lymphatic area. 


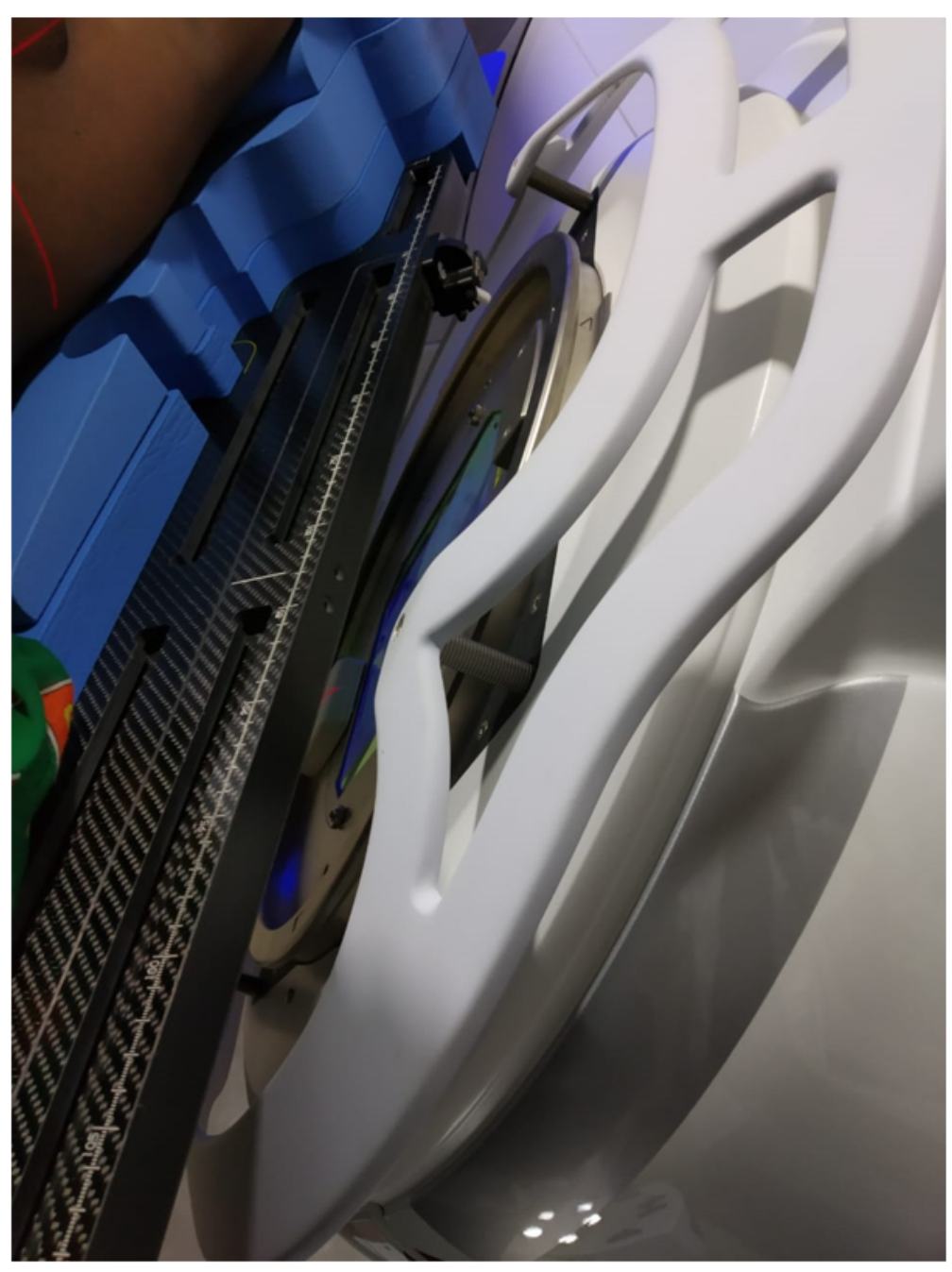

Figure 2

A closer look at the figure depicts impending collusion of gantry with left side (contralateral) of couch - while patient is immobilized on AlO breast and lung board mounted on base plate - after bringing the planning iso-centre to machine iso-centre by the way of shifting of couch in $x$-, $y$ - and $z$-axis as determined by TPS for iso-centric treatment of chest wall and draining lymphatic area. 


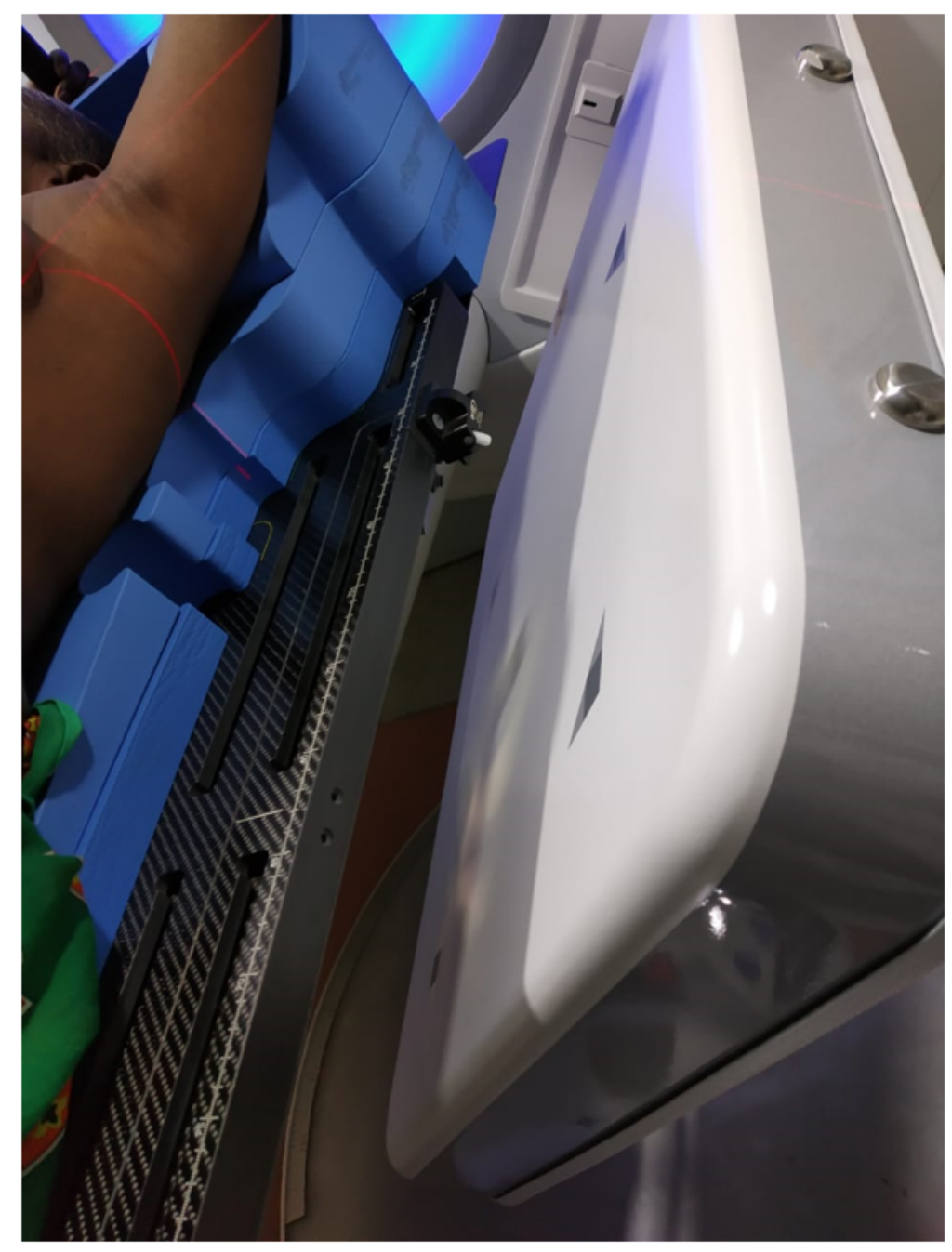

\section{Figure 3}

Flat panel kV x-ray detector barely passing the left lateral side of couch without collusion in one of the patient with thin body habitus planned for loco-regional irradiation after mastectomy 

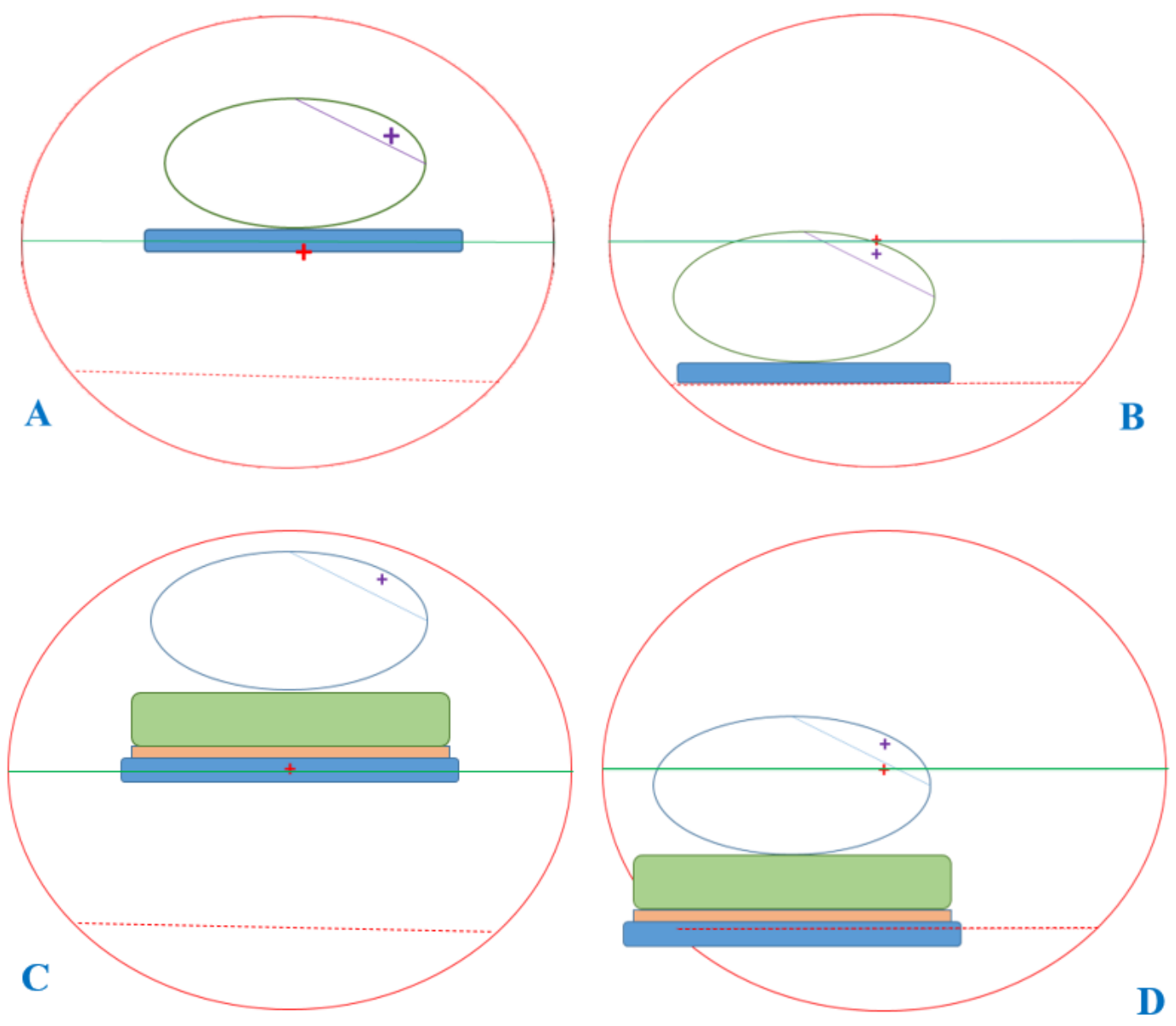

\section{Figure 4}

Pictorial illustration of hypothesis in geometrical terms that immobilizing patients on AIO breast and lung board leads to collusion of gantry with couch whose position is corrected for treatment after shifting the couch in $x-, y$-, and $z$-axes as determined by TPS for iso-centric post-operative local / loco-regional radiotherapy is supine position*, $\varphi$ A. Positioning of patients on base plate alone before shifting the couch in order to take planning isocentre (centre of CTV) to machine isocentre $B$. Position of couch after shifting the couch in 3 axes ( $x$-, $y$ - and $z$-axes) that is determined by TPS w.r.t patients positioned on base plate alone $\mathrm{C}$. Positioning of patients on AIO breast and lung board before shifting the couch in order to take planning isocentre (centre of CTV) to machine isocentre D. Position of couch after shifting the couch in 3 axes ( $x$ - $y$ - and $z$-axes) that is determined by TPS w.r.t patients positioned on AIO breast and lung board. In this figure, couch is seen to interrupt the circle of rotation of gantry around iso-centre that predict that gantry will collude with couch (whose position is corrected for implementation of VMAT) if it is rotated around $360^{\circ}$ around the machine isocentre. *Purple cross is planning isocentre, red cross is machine isocentre, blue line is chord of axial circumference of thorax representing radiotherapy target in case of carcinoma breast, Green line represents diameter of circle of rotation of gantry around the isocentre, red dashed line represent secant of circle of rotation of gantry around isocentre, blue circle represents axial section of thorax, blue rectangular slab represent couch, orange slab represents base plate, green slab represents AlO breast and lung board. $\Phi$ Blue and red cross actually superimpose in the clinical practice, in that planning iso-centre (centre of CTV) is brought to the machine iso-centre for all iso-centric treatment. Both these crosses are kept apart only for the purpose of pictorial illustration. 